\title{
Dual Frequency Comb Assisted Analog-to-Digital Conversion
}

\author{
Callum Deakin ${ }^{1}$ and Zhixin LiU ${ }^{1}$ \\ ${ }^{1}$ Optical Networks Group, Department of Electronic and Electrical Engineering, University College London, London, U.K. \\ *Corresponding author: callum.deakin.17@ucl.ac.uk; zhixin.liu@ucl.ac.uk
}

Compiled January 22, 2020

\begin{abstract}
Photonic analog to digital conversion offers promise to overcome the signal-to-noise ratio (SNR) and sample rate trade-off in conventional analog to digital converters (ADCs), critical for modern digital communications and signal analysis. We propose using phasestable dual frequency combs with a fixed frequency spacing offset to downconvert spectral slices of a broadband signal and enable high resolution parallel digitization. To prove the concept of our proposed method, we demonstrate the detection of a $10-\mathrm{GHz}$ subcarrier modulated (SCM) signal using 500-MHz bandwidth ADCs by optically converting the SCM signal to ten 1-GHz bandwidth signals that can be processed in parallel for full signal detection and reconstruction. Using sinusoidal wave based standard ADC testing, we demonstrate a spurious-free dynamic range (SFDR) of $>45 \mathrm{~dB}$ and signal-to-noise-and-distortion (SINAD) of $>20 \mathrm{~dB}$, limited by the receiver front-end design. () 2020 Optical Society of America
\end{abstract}

http://dx.doi.org/10.1364/ao.XX.XXXXXX

Analog to digital converters (ADCs) are essential to the acquisition and processing of time varying electrical signals, being the basis of real-time oscilloscopes and enabling signal analysis across a broad spectrum of scientific and engineering disciplines. In particular, the exponential growth of optical and wireless communications over the past few decades has driven demand for high speed and high resolution ADCs at greater than $10 \mathrm{GHz}$ bandwidth [1].

High speed ADCs followed by tailored digital signal processing (DSP) is the typical approach when attempting high resolution detection of broadband signals. State-of-the-art ADCs can achieve up to 7 bits effective number of bits (ENOB), which corresponds to about $44 \mathrm{~dB}$ signal-to-noise ratio (SNR) at $10 \mathrm{GHz}$, but this decays to around 5 bits when large bandwidths of over $70 \mathrm{GHz}$ are required [2]. The cause of this loss in resolution is twofold. Firstly, the clock jitter (or phase noise) requirements of the ADC's sampling clock increases with sampling frequency and therefore causes inevitable degradation of SNR at high sampling rates. Secondly, present semiconductor manufacturing limits the switching speed of the component transistors and introduces comparator ambiguity at high frequencies [3].
Furthermore, the utilization of advanced DSP such as deep learning algorithms for the processing of ADC captured signals is reliant on small parallelization overheard as processors approach their upper bounds of single threaded instructions per second due to thermal and quantum effects [4]. Thus, efficient serial to parallel conversion and data processing is required for for analysis of the captured data.

These challenges of high speed, high resolution signal detection as well as serial to parallel signal conversion have led to a variety of solutions across industry and academia. Leading instrument manufacturers have developed electronic time interleaving and digital/analogue bandwidth interleaving techniques to increase sampling rate and bandwidth [5]. More exotic solutions in academia include optical time stretching to improve ADC resolution and bandwidth [6, 7], and optical Fourier transforms to enhance the digital signal processing capability $[8,9]$.

Many of these methods attempt to down convert spectral slices of a high bandwidth signal so that they can be processed by low speed high resolution ADCs in parallel. Dual frequency combs are ideal for this purpose, being widely used in spectroscopy to down convert the optical response of a material to the low frequency RF domain [10], and have been further utilized for low-noise, low distortion spectral replication [11, 12].

In this paper, we propose using the dual frequency comb approach to down convert high bandwidth signals for high resolution parallel digitization. As a proof of concept, we optically downconvert a $10-\mathrm{GHz}$ bandwidth subcarrier modulated (SCM) signal, with each channel modulated with Nyquistshaped 4-level pulse amplitude modulation (PAM4) format, to ten 1-GHz bandwidth SCM-PAM4 signals using two frequency combs with stable phase and a fixed frequency offset of $1 \mathrm{GHz}$. The down-converted sub-band signals are captured using two 2.4-GSa/s high-resolution (14 bits) ADCs for balanced-detection of $500 \mathrm{MHz}$ baseband bandwidth. We achieve demodulation of the SCM-PAM4 signal of up to $20 \mathrm{~dB}$ SNR, limited by the ENOB of the digital to analog converter (DAC) used. Using standard ADC performance testing method, we obtained a spurious-free dynamic range (SFDR) of $45 \mathrm{~dB}$ across and a signal-to-noise-anddistortion (SINAD) of around $20 \mathrm{~dB}$, assuming $500 \mathrm{MHz}$ baseband bandwidth. To the best of our knowledge, this is the first demonstration of modulated signal detection using the dual comb method. Importantly, our work shows that the achievable SINAD is mainly limited by receiver front-end, which could be further improved for potentially significant improvement of the 


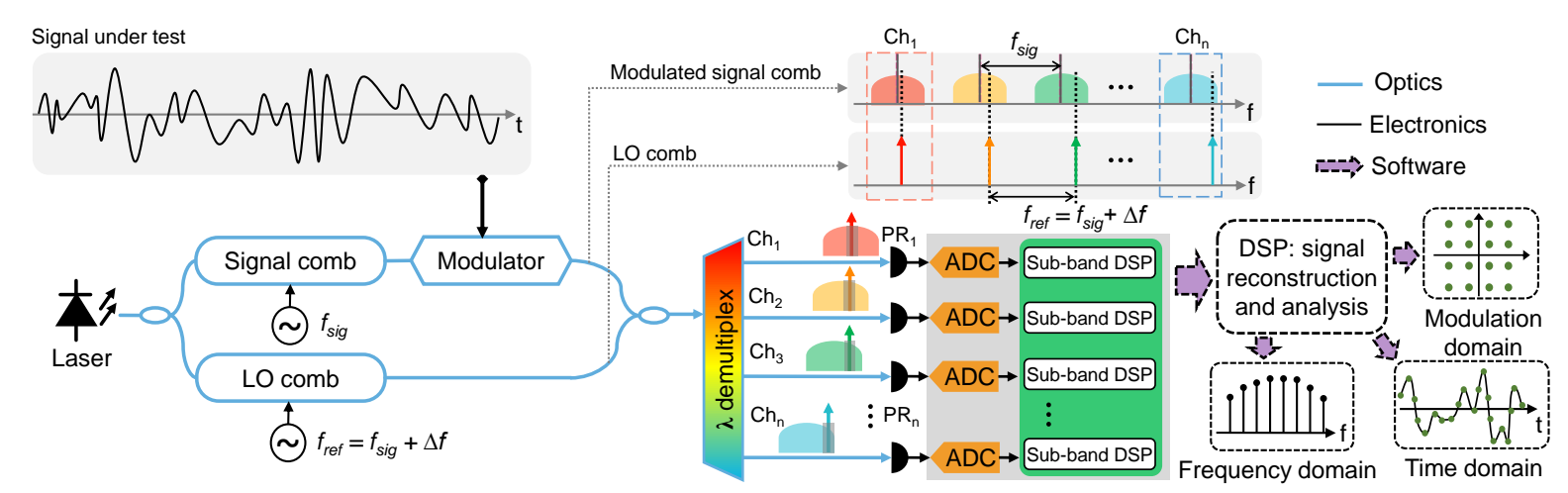

Fig. 1. A dual frequency comb assisted ADC. Beating between two frequency combs of spacing $f_{\text {sig }}$ and $f_{\text {ref }}$ can downconvert spectral slices of the signal under test to baseband with little distortion. These slices can then be detected at high resolution by a bank of parallel low speed ADCs. ADC: analog to digital converter; PR: photoreceiver; Ch: channel; DSP: digital signal processing.

system performance.

The schematic diagram of our proposed photonic ADC scheme is shown in Fig. 1. Two frequency combs with tone spacing of $f_{\text {sig }}$ and $f_{\text {ref }}$ are generated from the same seed laser source, which ensures that the combs are mutually coherent. The 'signal' comb $\left(f_{\text {sig }}\right)$ is modulated by driving a Mach-Zehnder modulator (MZM) with the signal under test and recombined with the 'local oscillator (LO)' $\left(f_{\text {ref }}=f_{\text {sig }}+\Delta f\right)$ comb before being demultiplexed by an arrayed waveguide grating (or other means of optical filtering) with a channel spacing of $f_{\text {sig. }}$.

When the $n$-th channel is incident on a photodiode, the beating between the $n$-th tone of the LO comb and the modulated $n$-th tone of the signal comb will generate a baseband signal centered at a frequency of $n \Delta f$. With an appropriate anti-aliasing filter, a bank of $n$ ADCs can therefore be used to interrogate $n$ $\Delta f$ spectral slices of the original signal under test. Importantly, the optical phase of the two frequency combs needs to be stable over the entire capturing period to facilitate waveform recovery.

There are several benefits to this approach. Firstly, the parallel detection of the sub-bands allows for digitizing high frequency components using low-speed but high-resolution ADCs. The whole signal spectrum can therefore be reconstructed at a resolution beyond the fundamental limit of a single wide-bandwidth ADC. Secondly, the optical spectral decomposition allows for parallel DSP of the sub-bands without any overhead for serial to parallel conversion, enabling reduced latency in the DSP chain and potential simplification of DSP hardware implementation. Thirdly, compared to digital/analog interleaving and time domain stretching techniques, our technique is not subject to the limitations imposed by sampling clock jitter since the bank of low speed ADCs are referenced from the same low frequency, low phase noise clock source. Finally, this approach is scalable: digitizing a signal of bandwidth $B$ via $N$ sub-bands requires $N$ comb lines with $\Delta f>B / N$, provided that $f_{\text {sig }} / 2>B$. Thus a high bandwidth signal can be processed simply by increasing the comb spacing $f_{\text {sig }}$ and by either increasing the number of channels $N$ or the sub channel bandwidth $\Delta f$. The only limitation to bandwidth therefore is being able to generate sufficient comb lines at a wide enough spacing to accommodate the signal under test. Considering recent demonstrations of ultra-wide band frequency combs with free spectral ranges of well over $100 \mathrm{GHz}[13,14]$, and in some cases up to $850 \mathrm{GHz}$ [15], our proposed photonic ADC approach has the potential to scale to several hundred $\mathrm{GHz}$ bandwidth. In comparison to electronic frequency interleaving, the use of optical combs as channelisers has the potential to avoid the need for highly accurate electronic filter design, which typically limits the performance of frequency interleaved ADCs [16].

The experimental setup is shown in Fig. 2. In our proof-ofconcept experiment, we generate both the signal comb and LO combs by driving cascaded phase and intensity modulators with sinusoidal RF signals at $26 \mathrm{GHz}$ and $27 \mathrm{GHz}$, respectively, generating 24 tones with $<3 \mathrm{~dB}$ power variation as in Ref. [17]. The combs were seeded with a narrow linewidth $(5 \mathrm{kHz})$ continuous wave (CW) signal amplified to $3 \mathrm{~W}$, resulting in an average power of $8 \mathrm{dBm}$ per tone and $>55 \mathrm{~dB}$ optical signal-to-noise ratio (OSNR). The signal comb was modulated by signal under test via an MZM biased at transmission null, which linearly mapped the electronic signals to all modulated tones to the optical domain with suppressed optical carriers. The peak-to-peak driving voltage was kept less than $0.3 V \pi$ to ensure a highly linear mapping from the electronic to the optical domain. The modulated signal comb has about $-15 \mathrm{dBm}$ per channel and was combined with the LO using a 50/50 coupler and subsequently filtered by two 30-GHz bandwidth tunable bandpass filters (BPFs) before detected by two 1.2-GHz photodiodes (PDs) followed by transimpedance amplifiers (TIA). By tuning the center wavelength of the BPFs, different sub-bands can be selected. The LO power was attenuated by $12 \mathrm{~dB}$ to avoid the saturation of the TIA used in this experiment. This attenuation can be avoided for a better SNR performance in practical systems, as we will discuss later.

The detected signals were captured by a pair of 14-bit 2.4 GSa/s ADCs arranged as a balanced detector to sequentially detect the $1 \mathrm{GHz}$ spectral slices by tuning the filters to the desired sub band. Full bandwidth detection of arbitrary waveforms can be realized by coherently detecting each sub-band and restitching them to a full-band signal via DSP. In this paper, we prove the concept by detecting a series of sub-band limited amplitude modulated signals. The fiber lengths of the two arms (signal comb and LO comb) were matched in our system and are attached to each other to minimize the impact from environment, ensuring optical phase only slowly drifted with temperature, allowing for the detection of the combined signals with stable optical phase over the entire period $(2.048 \mu \mathrm{s})$ of the SCM-PAM4 signals. Any implementation will require phase stability for at least the length of the capture duration, the techniques for which are well established [18, 19].

As shown in the insets in Fig. 2, the signal under test was generated by using either a 6-bit 32-GSa/s digital-to-analog converter (DAC) for the generation of the subcarrier modulated 


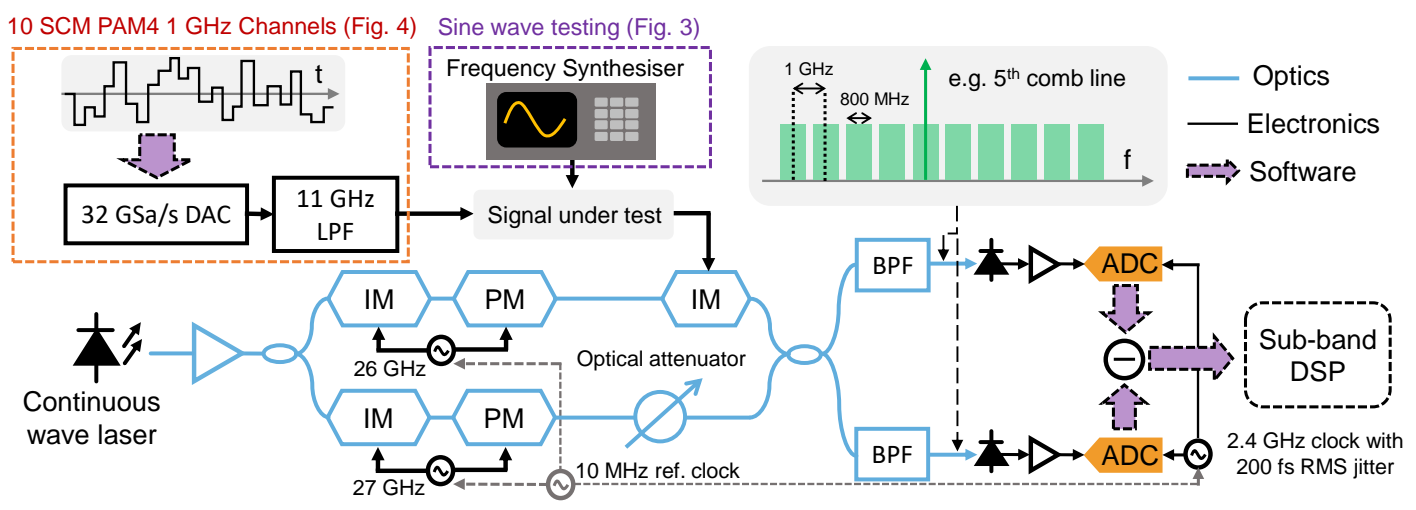

Fig. 2. Experimental setup. Two $26 \mathrm{GHz}$ tunable bandpass filters are used to select desired $1 \mathrm{GHz}$ sub-band. BPF: bandpass filter, LPF: low pass filter, DAC: digital to analog converter, IM: intensity modulator, PM: phase modulator.

(SCM) signals, or a frequency synthesizer to generate a range of single frequency sine wave for SFDR and SINAD testing based on the IEEE ADC testing standard [20]. In the DAC setup, a $11 \mathrm{GHz}$ low pass filter was used to remove the aliases. The RF driving power was kept at $0 \mathrm{dBm}$ for optimum performance. The SCM signal under test was a 10 channel, $1 \mathrm{GHz}$ spaced, 800 MBaud sub carrier modulated Nyquist-shaped PAM-4 signal. The SCM modulation format allows for independent detection and decoding of each subchannel without requiring parallel detection of the subbands. A $40 \mathrm{MHz}$ frequency shift was added to the baseband of each channel to avoid the low frequency filtering (DC-10 MHz) caused by the AC-coupled post detection electrical amplifier used in our experiment. This allows for an assessment of the system performance without the significant investment of acquiring sufficient ADCs to detect a signal with a full bandwidth baudrate. A limitation of this sequentially detected experiment is that we cannot evaluate the performance impact of any channel mismatch, which can be compensated through calibration and the channel restitching DSP [21]. The signal demodulation and analysis was digitally processed offline, using a two sample-per-symbol 17-tap feed-forward equalizer to equalize the frequency roll-off within the sub-band. Finally, for performance evaluation, we also captured the SCM-PAM4 directly from the DAC output using a Tektronix DPO72304DX digital phosphor oscilloscope with a sampling rate of $100 \mathrm{GSa} / \mathrm{s}$ and a $3 \mathrm{~dB}$ analog bandwidth of $23 \mathrm{GHz}$.

We first investigate the system performance using sinusoidal waves based on the IEEE standard. Fig. 3(a) shows the SFDR and the SINAD, as well as the effective number of bits, $\mathrm{ENOB}=(\mathrm{SINAD}-1.76) / 6.02$. The SFDR remains consistently above $45 \mathrm{~dB}$ and the SINAD above $20 \mathrm{~dB}$ for almost the entire bandwidth, with both figures of merit experiencing a gradual roll off of around $3 \mathrm{~dB}$ across the measurement bandwidth. This decrease in performance with higher frequency can mainly be attributed to the high frequency-roll off from 0.1 to $10 \mathrm{GHz}$ in the electrical signal chain (inc. RF cables and connectors), which was about $3 \mathrm{~dB}$ in our system. Since the optical bandwidth of each subchannel is $1 \mathrm{GHz}$, only $500 \mathrm{MHz}$ electronic base band bandwidth is needed for the signal detection. Thus, the SINAD was calculated by integrating the noise and distortion power from 0-500 MHz.

Fig. $3 \mathrm{~b}$ shows the RF spectrum calculated from the captured subcarrier signals when the signal under test was a $5.25 \mathrm{GHz}$ sinusoidal signal. The BPFs were tuned to select the 5 th subchannel (4.5-5.5 GHz), which is displayed as an average of
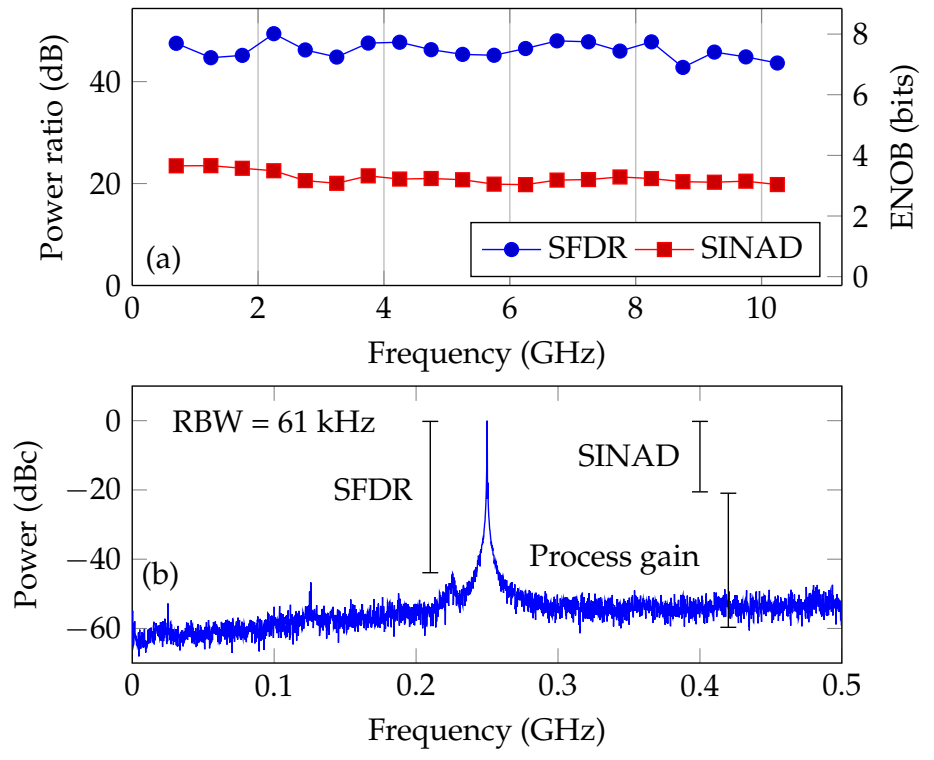

Fig. 3. (a) SFDR and SINAD/ENOB as a function of frequency up to $10.5 \mathrm{GHz}$, with (b) an example fast fourier transform (FFT) showing a $5.25 \mathrm{GHz}$ signal detected in the $4.5-5.5 \mathrm{GHz}$ sub-band. All values are calculated over a $1 \mathrm{GHz}$ range after digital Nyquist filtering with an average of 4 16384-point FFTs. The rise of noise floor with increased frequency is attributed to the receiver amplifier.

four 16384-point FFTs corresponding to an FFT process gain of $39.1 \mathrm{~dB}$. Note that because we do not perform full coherent detection of the sub-band, the two halves of each $1 \mathrm{GHz}$ subband are aliased into the same $500 \mathrm{MHz}$ half sub-band. Thus a $4.75 \mathrm{GHz}$ signal will appear as the same $250 \mathrm{MHz}$ sub-band signal as the 5.25 Ghz signal shown in Fig. 3(b).

A limitation of our current system, which limits performance compared to other photonic ADC designs (e.g. Ref. [7] with 7 bits ENOB at $41 \mathrm{GHz}$ ), can be illustrated by analyzing Fig. 3(b). The SFDR is limited by the presence of spurs caused by receiver-side electrical amplifiers. Furthermore, the used PD-TIA saturated at a low input optical power of $-13 \mathrm{dBm}$, which prevented the full use of the ADCs dynamic range and a significant drop in the measured SINAD. This is highlighted by the large disparity between the measured SFDR and SINAD. The above-mentioned engineering problems can be resolved with the optimization of 
the receiver front-end design, such as the transimpedance and the saturation power of the TIA as well as the 2nd stage voltage amplifier gain to obtain a potentially significant improvement (e.g. a minimum of $12 \mathrm{~dB}$ from higher saturation power) in the SINAD and ENOB.
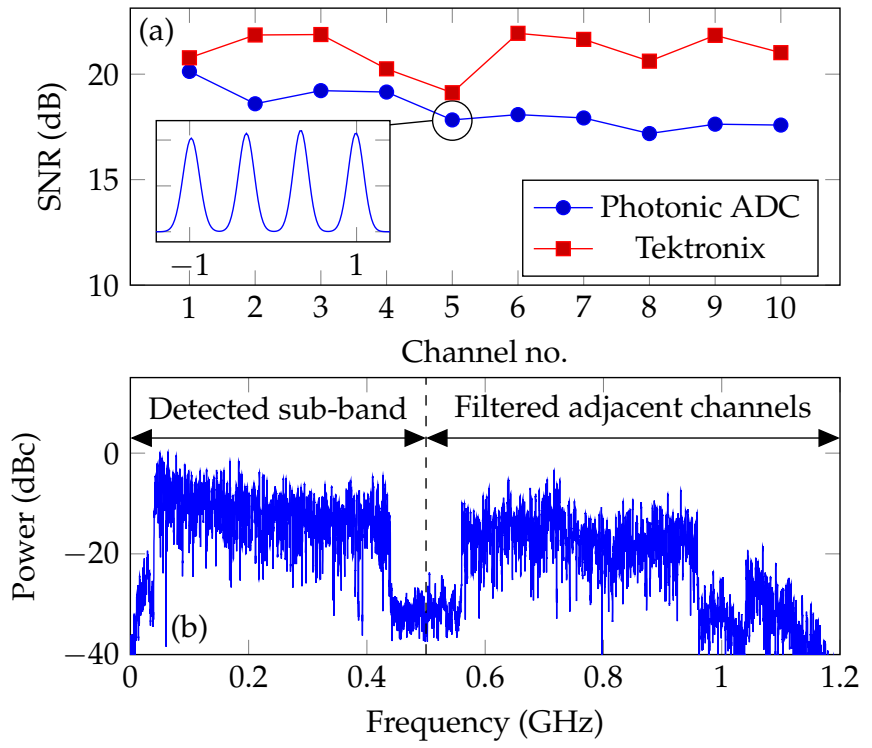

Fig. 4. 10 channel SCM PAM4 detection performance with $1 \mathrm{GHz}$ sub-bands. (a) SNR vs channel number for our photonic ADC and a Tektronix DPO72304DX 100 GSa/s 23 GHz oscilloscope, with example sample histogram inset from the 5 th channel. (b) Example sub-band spectrum from the 5th sub-band showing the target 5 th channel and adjacent channels across the full ADC bandwidth.

The measured SNR values for the 10-GHz bandwidth SCMPAM4 signal are shown in Fig. 4(a), with an example sampling histogram inset from which the SNR values are derived. The highest SNR we obtained was $20.1 \mathrm{~dB}$ in the first channel (0.5-1.5 GHz). An increase in channel number shows a decrease in SNR, with the final 10th channel (at $10 \mathrm{GHz}$ ) suffering a $2.5 \mathrm{~dB}$ SNR penalty with reference to the first channel. This SNR trend agrees with that demonstrated for SINAD/ENOB in Fig. 3(a), with a further penalty of approximately $3 \mathrm{~dB}$ to the absolute SNR due to inter-channel interference, since transmitting a single subband from the DAC allows us to recover the higher SNR/SINAD demonstrated in Fig. 3(a). In addition, the achievable SNR in this experiment was fundamentally limited by the ENOB of the DAC used to produce the SCM signal, which is highlighted by the comparison with the conventional Tektronix DPO72304DX oscilloscope. The maximum achievable SNR with direct detection by the oscilloscope is around $22 \mathrm{~dB}$ (3.4 ENOB) which is in line with our previous experience of the DAC perfomance. Noticeably, the capture with the oscilloscope does not experience any significant SNR roll off as the oscilloscope noise floor is $10-\mathrm{dB}$ below that of the DAC.

Fig. 4(b) shows the spectrum of the received sub-band signal. Visible is the target 5th channel as well as the adjacent channels that are filtered out digitally, with further channels suppressed by the ADC anti-aliasing filter who effects can be seen above $1 \mathrm{GHz}$. The strong $(8 \mathrm{~dB})$ roll off of the channel of interest is a result of the frequency response of the detector and is corrected in this experiment by the previously described equalizer.
In summary, we propose an analog to digital conversion method based on phase-stable dual frequency combs. Our system down converts a sub-band of a broadband signal optically for detection with high-resolution ADCs, promising potential to overcome the resolution and bandwidth tradeoff in the conventional time-interleaving ADC paradigm. The feasibility of the proposed method was experimentally demonstrated by detection of a 10-GHz SCM-PAM4 signal with 500-MHz bandwidth ADCs, showing 20dB SNR that is only limited by the signal generator in our experiment. We identify that the moderate SFDR and SINAD performance in our current system is caused by the receiver front-end, and that practical implementations will require restitching of the detected sub-channels via DSP and an active phase stability mechanism. Our proposed method has the potential to reduce the susceptibility to timing jitter compared to photonic and electronic time interleaving ADCs, and does not rely on the highly accurate filter bank design required for electronic frequency interleaving.

Funding. EPSRC grant (EP/R041792/1), EPSRC programme grant TRANSNET (EP/R035342/1), Royal Society Paul Instrument Fund (PIF/R1/180001).

Disclosures. The authors declare no conflicts of interest.

\section{REFERENCES}

1. B. Murmann, "ADC performance survey 1997-2019," http://web. stanford.edu/ murmann/adcsurvey.html. Accessed: 2019-11-12.

2. Keysight, "Infiniium UXRSeries Oscilloscopes," .

3. R. H. Walden, IEEE J. on selected areas communications 17, 539 (1999).

4. I. L. Markov, Nature 512, 147 (2014).

5. T. Drenski and J. C. Rasmussen, "ADC \& DAC Technology trends and steps to overcome current limitations," in 2018 OFC, (2018), p. M2C.1.

6. J. K. Fischer et al., J. Light. Technol. 29, 378 (2011).

7. A. Khilo et al., Opt. Express 20, 4454 (2012).

8. D. Hillerkuss et al., Opt. Express 18, 9324 (2010).

9. H. Hu et al., "Comb-assisted real-time Discrete Fourier Transform processor," in 2017 OFC, (2017), p. M2J.3.

10. I. Coddington, N. Newbury, and W. Swann, Optica 3, 414 (2016).

11. V. Ataie, D. Esman, B. P.-P. Kuo, N. Alic, and S. Radic, Science 350, 1343 (2015).

12. D. J. Esman, V. Ataie, B. P. Kuo, E. Temprana, N. Alic, and S. Radic, J. Light. Technol. 34, 5669 (2016).

13. M. A. Foster, J. S. Levy, O. Kuzucu, K. Saha, M. Lipson, and A. L. Gaeta, Opt. Express 19, 14233 (2011).

14. Y. Okawachi, K. Saha, J. S. Levy, Y. H. Wen, M. Lipson, and A. L. Gaeta, Opt. Lett. 36, 3398 (2011).

15. P. Del'Haye, T. Herr, E. Gavartin, R. Holzwarth, and T. J. Kippenberg, Phys. Rev. Lett. 107, 063901 (2011). ArXiv: 0912.4890.

16. D. Asemani and J. Oksman, "Influences of oversampling and analog imperfections on Hybrid Filter Bank A/D converters," in 2006 49th IEEE International Midwest Symposium on Circuits and Systems, , vol. 1 (2006), pp. 123-126.

17. V. Torres-Company, J. Lancis, and P. Andrés, Opt. Lett. 33, 1822 (2008).

18. Z. Liu, J. Kakande, B. Kelly, J. OCarroll, R. Phelan, D. J. Richardson, and R. Slavík, Nat. communications 5, 5911 (2014).

19. Z. Chen, M. Yan, T. W. Hänsch, and N. Picqué, Nat. communications $\mathbf{9}$, 3035 (2018).

20. IEEE Std 1241-2010 (Revision IEEE Std 1241-2000) pp. 1-139 (2011).

21. L. Guo, S. Tian, Z. Wang, K. Yang, and L. Qiu, Circuits, Syst. Signal Process. 33, 3697 (2014). 


\section{FULL REFERENCES}

1. B. Murmann, "ADC performance survey 1997-2019,", http://web. stanford.edu/ murmann/adcsurvey.html. Accessed: 2019-11-12.

2. Keysight, "Infiniium UXRSeries Oscilloscopes," .

3. R. H. Walden, "Analog-to-digital converter survey and analysis," IEEE J. on selected areas communications 17, 539-550 (1999).

4. I. L. Markov, "Limits on fundamental limits to computation," Nature $\mathbf{5 1 2}$ 147-154 (2014).

5. T. Drenski and J. C. Rasmussen, "ADC \& DAC Technology trends and steps to overcome current limitations," in 2018 OFC, (2018), p. M2C.1.

6. J. K. Fischer et al., "High-Speed Digital Coherent Receiver Based on Parallel Optical Sampling," J. Light. Technol. 29, 378-385 (2011).

7. A. Khilo et al., "Photonic ADC: overcoming the bottleneck of electronic jitter," Opt. Express 20, 4454-4469 (2012).

8. D. Hillerkuss et al., "Simple all-optical FFT scheme enabling Tbit/s real-time signal processing," Opt. Express 18, 9324-9340 (2010).

9. H. Hu et al., "Comb-assisted real-time Discrete Fourier Transform processor," in 2017 OFC, (2017), p. M2J.3.

10. I. Coddington, N. Newbury, and W. Swann, "Dual-comb spectroscopy," Optica 3, 414 (2016).

11. V. Ataie, D. Esman, B. P.-P. Kuo, N. Alic, and S. Radic, "Subnoise detection of a fast random event," Science 350, 1343-1346 (2015).

12. D. J. Esman, V. Ataie, B. P. Kuo, E. Temprana, N. Alic, and S. Radic, "Detection of Fast Transient Events in a Noisy Background," J. Light. Technol. 34, 5669-5674 (2016).

13. M. A. Foster, J. S. Levy, O. Kuzucu, K. Saha, M. Lipson, and A. L. Gaeta, "Silicon-based monolithic optical frequency comb source," Opt. Express 19, 14233-14239 (2011).

14. Y. Okawachi, K. Saha, J. S. Levy, Y. H. Wen, M. Lipson, and A. L. Gaeta, "Octave-spanning frequency comb generation in a silicon nitride chip," Opt. Lett. 36, 3398 (2011).

15. P. Del'Haye, T. Herr, E. Gavartin, R. Holzwarth, and T. J. Kippenberg, "Octave Spanning Frequency Comb on a Chip," Phys. Rev. Lett. 107, 063901 (2011). ArXiv: 0912.4890.

16. D. Asemani and J. Oksman, "Influences of oversampling and analog imperfections on Hybrid Filter Bank A/D converters," in 2006 49th IEEE International Midwest Symposium on Circuits and Systems, , vol. 1 (2006), pp. 123-126.

17. V. Torres-Company, J. Lancis, and P. Andrés, "Lossless equalization of frequency combs," Opt. Lett. 33, 1822 (2008).

18. Z. Liu, J. Kakande, B. Kelly, J. OCarroll, R. Phelan, D. J. Richardson, and R. Slavík, "Modulator-free quadrature amplitude modulation signal synthesis," Nat. communications 5, 5911 (2014).

19. Z. Chen, M. Yan, T. W. Hänsch, and N. Picqué, "A phase-stable dualcomb interferometer," Nat. communications 9, 3035 (2018).

20. "leee standard for terminology and test methods for analog-to-digital converters," IEEE Std 1241-2010 (Revision IEEE Std 1241-2000) pp. 1-139 (2011).

21. L. Guo, S. Tian, Z. Wang, K. Yang, and L. Qiu, "Analysis of Channel Mismatch Errors in Frequency-Interleaved ADC System," Circuits, Syst. Signal Process. 33, 3697-3712 (2014). 